\title{
Method for the recovery of images in databases of skin cancer
}

\section{Autores}

Amelec Viloria, Noel Varela, Narledys Nuñez-Bravo, Omar Bonerge Pineda Lezama

\section{Abstract}

Deep learning is widely used for the classification of images since the ImageNet competition in 2012 (Zaharia et al. in Common ACM 59(11):56-65, 2016, [1] ; Tajbakhsh et al. in IEEE Trans Med Imaging 35(5):1299-1312, 2016, [2] ). This image classification is very useful in the field of medicine, in which there is a growing interest in the use of data mining techniques in recent years. In this paper, a deep learning network was selected and trained for the analysis of a set of skin cancer data, obtaining very satisfactory results, as the model surpassed the classification results of trained dermatologists using a dermatoscope, other automatic learning techniques, and other deep learning techniques.

Palabras clave:

Deep learning, Medical images, Clinical data analysis. 